\title{
An analog-to-digital converter for on-line use in response dynamics research*
}

\author{
PREDRAG VRTUNSKI \\ Cleveland Psichiatric Institute \\ 1708 Aiken Avenue, Cleveland, Ohio 44109
}

A general-purpose translator was designed for an analysis of the dynamic properties of the intracranially reinforced (ICR) barpressing response. It is used principally as an animal training device.

The purpose of this paper is to describe a special-purpose translator designed for an analysis of the dynamic properties of the intracranially reinforced (ICR) barpressing response. The requirements for the translator are determined mainly by the nature of the response. In the case of response dynamics analysis, an individual barpressing response is defined in terms of a force distribution as a function of time (Notterman \& Mintz, 1965). Thus, instead of an event mark or a step increment on a cumulative recorder, the response is recorded as a complex curve, i.e., the voltage analog of the emitted force. To reduce complexity to a limited number of numerical indices, a set of reference points along the force distribution is needed. These reference points are defined by two force levels, threshold and criterion (Fig. 1). In this manner, the translator duplicates the hysteresis characteristics of a microswitch. The translator also provides reference points between which various response parameters can be measured by additional equipment: response duration $\left(\mathrm{X}_{1} \rightarrow \mathrm{X}_{2}\right)$, interresponse time $\left(X_{2} \rightarrow X_{1}\right)$, time interval needed to reverse the muscle action from the paw extension to paw flexion $\left(\mathrm{Y}_{1} \rightarrow \mathrm{Y}_{2}\right)$ or to release the bar $\left(\mathrm{Y}_{2} \rightarrow \mathrm{X}_{2}\right)$. To measure the peak force of the response, the highest value reached within the $Y_{1} \rightarrow Y_{2}$ interval has to be retained (Vrtunski, Murray, \& Wolin. in press). These reference points also serve for setting the reinforcement contingencies. Thus, response dynamics is significantly different when the reinforcing stimulus onset coincides with $Y_{1}$ and when it coincides with the $X_{2}$ point along the force distribution curve (VItunski, 1972).

Operation of the translator (Fig. 2) is as follows. The animal's barpress is transmitted by a pressure transducer. The analog signal from the transducer is amplified and fed into a sample-and-hold module and analog-to-digital (A-D) converter (10-bit conversion). A-D conversion rate is determined by the setting of the clock. The clock control consists of several delay and monostable multivibrator circuits. so that proper synchronization can be maintained (Fig. 3A). Thus, the hold mode of the sample-and-hold module takes into account the

*The described device was constructed for a study supported by Grant GRS-05563 from the National Institutes of Health. acquisition time of that module before the sync pulse to the A-D converter is issued. The typical $10-\mu \mathrm{sec}$ pulse of $\mathrm{K}$ series circuits is shortened and the slope of the leading edge reduced to $200 \mathrm{nsec}$ by feeding the delay clock pulse through a multivibrator and a double NAND gate (Fig. 3B). Also, updating of the storage register requires a $12-15-\mu$ sec delay after conversion sync pulse.

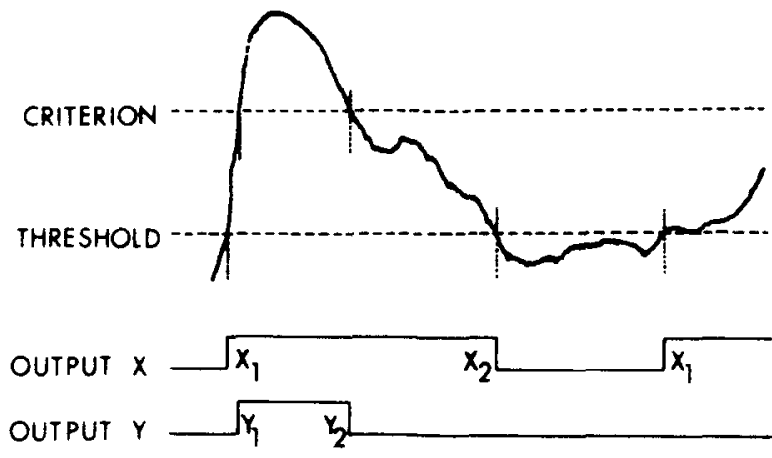

Fig. 1. Upper curve represents a diagram of the barpressing response in a self-stimulating rat. Increase in amplitude corresponds to increase in force exerted upon the bar. There are two force levels set in thumbwheel switches of the translator, the threshold and the criterion. These reference points determine the force levels at which Outputs $X$ and $Y$, from two sets of digital comparators, respectively, change their status.

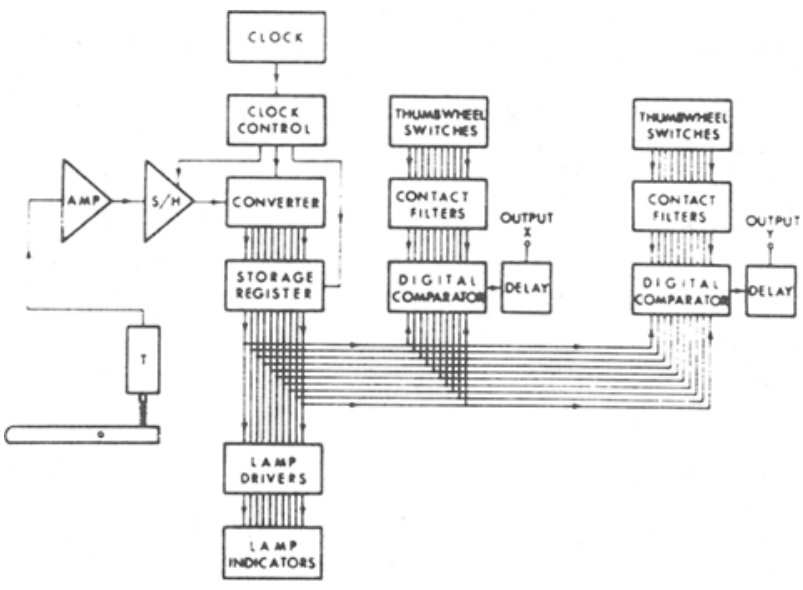

Fig. 2. Block diagram of the response translator. T. pressure transducer. Statham Type UC 2: AMP. operational amplifier. Analogic Devices Type 144K; S/H, sample-and-hold module. Teledyne-Philbric Type PPT\&H: A-D converter, Digital Equipment Corporation (DEC) Type A811: clock, 3/3 of DEC Type K303 time delays (numerator indicates number of circuits used. and denominator, number of circuits in a single module): storage register, Analogic Type MP 1612-SR: lamp drivers. 10/8 DEC Type K683: thumbwheel switches. Chicago Dynamic Industries, Type TTB: contact filters, 10/8 DEC Type K581: digital comparators, 10/4 DEC Type K 174: delay. 1/3 DEC Type K 303. The last four components refer to one output only. 

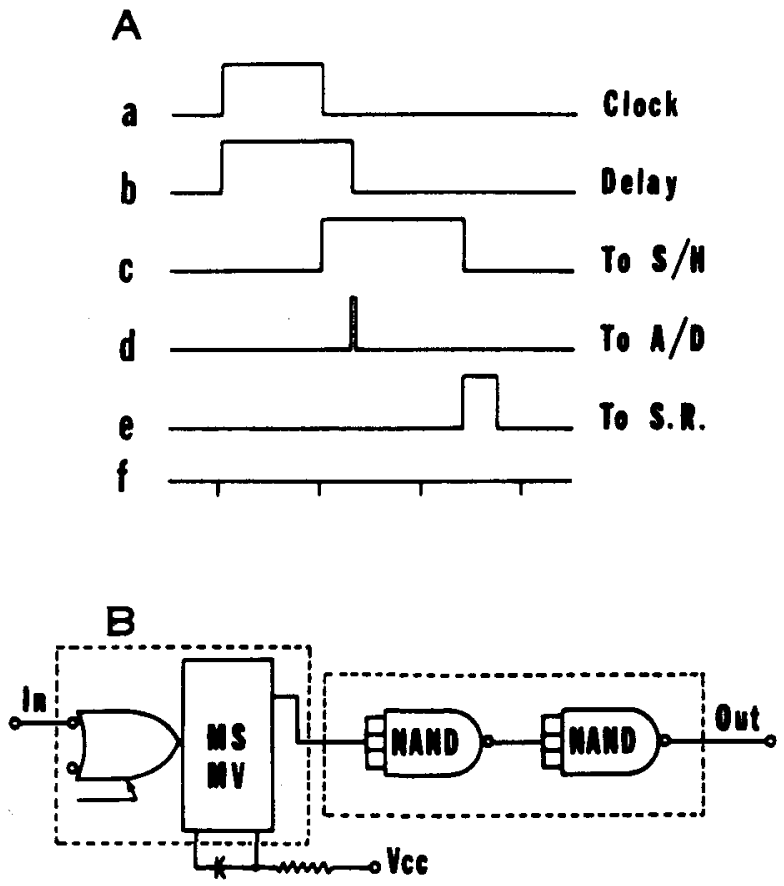

Fig. 3. A: Pulse configuration within clock control necessary for synchronized A-to-D conversion. Clock pulse (a) is the only input into the control. Delay pulse (b) is used for triggering 300-nsec sync (d) to A/D converter (Diagram B). Pulse to $S / H$ module (c) "holds" the analog signal "still" during conversion. Pulse to storage register (e) updates the register with digital value of the preceding conversion. Time marks (f), $10 \mu \mathrm{sec}$. B: A monostable multivibrator (Motorola Type MC 851P) and a double NAND gate (Motorola Type MC 450) used to reduce $10-\mu$ sec pulse to a 300 -nsec pulse.

The conversion rate used in our work is $2 \mathrm{KHz}$ (A811 has the sampling rate capability of $50 \mathrm{KHz}$ ). The

converted analog signal is stored by storage register and fed into two sets of digital comparators and a visual display of the binary value of the bar pressure. Output of each set of comparators depends upon comparison between a fixed digital value set with thumbwheel switches and the amplitude of the barpressing response. As long as the bar pressure is lower than the given thumbwheel-switch value, the output of the comparators stays low. Once that value is exceeded, the output goes high. In order to prevent transient changes in comparator levels due to updating of the storage register, the output of the comparators is passed through a delay.

By loading the bar with known weights, the translator is calibrated. With maximal sensitivity of the input amplifier, a unit increment on the thumbwheel switches corresponds to approximately $0.109 \mathrm{~g}$ of static equivalent pressure.

The described response translator is a highly accurate, fast, and flexible tool. It can be used as an A-D converter for a number of laboratory measurements. As presented, it is used for animal training purposes. To obtain a permanent record, or to time durations between set force levels, additional equipment must be interfaced, a principal limitation of the device.

\section{REFERENCES}

Notterman, J. M., \& Mintz, D. E. Dynamics of response, New York: Wiley, 1965

Vrtunski, $P$. Components of the intracranially reinforced barpressing response. Presented at the Society for Neuroscience meeting, Houston, Texas, October 1972 (abstract).

Vrtunski, P., Murray, R., \& Wolin, L. R. Alcohol effect on intracranially reinforced response. Quarterly Journal of Studies on Alcohol, in press.

(Received for publication December 15, 1972; revision received February 5, 1973.) 